\title{
Outcome of Pandemic H1N1 Pneumonia: Clinical and Radiological Findings for Severity Assessment
}

\author{
Woo Hyun Cho', Yun Seong Kim', Doo Soo Jeon', Ji Eun Kim', Kun II Kim², Hee Yun Seol, Ki Uk Kim³, \\ Hye Kyung Park ${ }^{3}$, Min Ki Lee ${ }^{3}$, Soon Kew Park ${ }^{3}$, and Yeon Joo Jeong ${ }^{4}$
}

Departments of ${ }^{1}$ Internal Medicine and ${ }^{2}$ Radiology, Pusan National University Yangsan Hospital, Yangsan; Departments of ${ }^{3}$ Internal Medicine and ${ }^{4}$ Radiology, Pusan National University Hospital, Busan, Korea

Background/Aims: Pandemic influenza A (H1N1) virus infection presents with variable severity. However, little is known about clinical predictors of disease severity. We studied the clinical predictors of severe pandemic H1N1 pneumonia and their correlation with radiological findings.

Methods: We reviewed medical and radiological records of adults with pandemic H1N1 pneumonia. After classification of patients into severe and non-severe groups, the following data were evaluated: demographic data, pneumonia severity index (PSI), CURB65, risk factors, time to first dose of antiviral medication, routine laboratory data, clinical outcome, and radiological characteristics.

Results: Of 37 patients with pandemic H1N1 pneumonia, 12 and 25 were assigned to the severe and non-severe groups, respectively. PSI score, serum aspartate aminotransferase (AST), alanine aminotransferase (ALT), and lactate dyhydrogenase $(\mathrm{LDH})$ levels were higher in the severe group than in the non-severe group $(p=0.035,0.0003,0.0023$, and 0.0002 , respectively). AST, ALT, and LDH levels were positively correlated with the radiological findings $(p<0.0001$, 0.0003 , and $<0.0001$, respectively) and with the number of involved lobes ( $p=0.663,0.0134$, and 0.0019 , respectively). The most common finding on high resolution computed tomography (HRCT) scans was ground-glass attenuation with consolidation $(n=22,60 \%)$, which had a predominantly patchy distribution $(n=31)$.

Conclusions: We demonstrated a positive correlation between clinical findings, such as serum AST, ALT, and LDH levels, and radiological findings. A combination of clinical and HRCT indicators would be useful in predicting the clinical outcome of pandemic H1N1 pneumonia. (Korean J Intern Med 2011;26:160-167)

Keywords: Influenza A virus, H1N1 subtype; Pneumonia; Severity of illness index

\section{INTRODUCTION}

Since the beginning of the 2009 influenza A (H1N1) pandemic, the cumulative number of infected people has increased rapidly [1,2]. Pneumonia has been reported as the major cause of hospitalization and death in confirmed cases of H1N1 [3-6]. However, there are limited data on the clinical predictors of severity and radiological features of pandemic H1N1 pneumonia.
Delayed antiviral treatment, severe hypoxemia, and multisystem organ failure have been reported to be significant negative prognostic factors among critically ill patients with pandemic $\mathrm{H} 1 \mathrm{~N} 1$ infection [7-11], while early diagnosis and assessment of high risk are likely to be important for successful treatment. It has been a challenge to differentiate pandemic H1N1 pneumonia from community-acquired pneumonia of bacterial origin or from pulmonary disease occurring from other causes.

Received: August 12, 2010

Revised : November 4, 2010

Accepted: November 17, 2010 
Thus, it is important to understand the clinical and radiological features of pandemic $\mathrm{H} 1 \mathrm{~N} 1$ pneumonia to facilitate early diagnosis and assessment of risk. The aim of this study was to find clinically useful predictors of severity and radiological features characteristic of pandemic $\mathrm{H} 1 \mathrm{~N} 1$ pneumonia. We studied the clinical predictors of the severe form of pandemic $\mathrm{H} 1 \mathrm{~N} 1$ pneumonia and explored the correlation of these predictors with high resolution computed tomography (HRCT) findings.

\section{METHODS}

\section{Patient selection criteria}

Thirty-seven adults who presented with confirmed pandemic $\mathrm{H} 1 \mathrm{~N} 1$ pneumonia at the Pusan National University Hospital and Pusan National University Yangsan Hospital, Korea, between August 1, 2009 and November 30, 2009 were recruited. Pandemic H1N1 infection was confirmed by a real-time RT-PCR assay performed on specimens aspirated from the nasopharyngeal or endotracheal tubes. Specimens were collected at the time of admission. Pneumonia was defined as the presence of acute respiratory symptoms and parenchymal lesions in the HRCT scan, which was performed at the time of admission. Patients who did not undergo the HRCT scan or were below 18 years old were excluded.

Patients were classified into two groups according to the clinical severity of the disease at the time of presentation: severe and non-severe groups. The severe group included; 1) patients requiring mechanical ventilation, 2) those requiring intravenous infusion of inotropic medication, or
3) those with a $\mathrm{PaO}_{2} / \mathrm{FiO}_{2}$ ratio $\leq 250$ [12].

This study was approved by the Institutional Review Board of Pusan National University Hospital.

\section{Clinical data}

We performed a retrospective review of the medical charts and radiological and laboratory records to obtain demographic data, pneumonia severity index (PSI) scores [13], CURB65 [14], comorbidity, time to first dose of antiviral medication, routine laboratory data, clinical outcome, and radiological characteristics.

\section{Image interpretation}

Six locations in each patient (right upper lobe, right middle lobe, right lower lobe, left upper lobe, lingular segment, left lower lobe) were evaluated for the presence of lung lesions and other abnormal findings. The computed tomography (CT) images were reviewed independently by two experienced chest radiologists who reached a consensus regarding the findings and extent of the disease. The CT scans were evaluated retrospectively for consolidation, air-bronchogram, ground-glass attenuation (GGA), GGA with intralobular reticular opacity ("crazypaving” appearance), centrilobular nodules, bronchial wall thickening, interlobular septal thickening, pleural effusion, and hilar or mediastinal lymph node enlargement. Laterality (unilateral or bilateral) and the number of involved lobes of lung lesions were also analyzed. The visual analysis on the extent of consolidation and GGA on the CT image was subjectively performed using a 10\% scale.

\section{Table 1. Demographic and clinical findings}

\begin{tabular}{lcccc}
\hline Variables & $\begin{array}{c}\text { Total } \\
(\mathrm{n}=37)\end{array}$ & $\begin{array}{c}\text { Severe group } \\
(\mathrm{n}=12)\end{array}$ & $\begin{array}{c}\text { Non-severe group } \\
(\mathrm{n}=25)\end{array}$ & $p$ value \\
\hline Mean age, yr (range) & $46.1 \pm 17.3(18-79)$ & $46.3 \pm 18.4(20-62)$ & $46.0 \pm 15.4(18-79)$ & 0.867 \\
Male & $21(57)$ & $6(50)$ & $15(60)$ & 0.5654 \\
Comorbidity & $18(49)$ & $8(67)$ & $10(40)$ & 0.1287 \\
CURB65 & $0.65 \pm 0.97$ & $1.00 \pm 1.12$ & $0.48 \pm 0.87$ & 0.1601 \\
Pneumonia severity index & $60.0 \pm 39.0$ & $83.4 \pm 45.7$ & $48.9 \pm 30.4$ & 0.035 \\
Time to antiviral treatment, day & $5.27 \pm 2.95$ & $6.41 \pm 4.44$ & $4.72 \pm 1.77$ & 0.2675 \\
Shock & 4 & 4 & 0 & \\
Acute respiratory distress syndrome & 5 & 5 & 0 & \\
Mechanical ventilation & 8 & 8 & 0 & \\
Death & 5 & 5 & 0 & \\
\hline
\end{tabular}

Values are presented as mean \pm SD or number (\%). 


\section{Statistical analysis}

The Mann-Whitney $U$ test was used to analyze continuous variables. Categorical variables were compared using the chi-squared test or Fisher's exact test. The Pearson correlation coefficient was used to evaluate correlations between clinical variables and the degree of radiological involvement. Statistical analyses were performed using the SAS version 9.1 (SAS Inc., Cary, NC, USA). In all analyses, $p<0.05$ (two-sided) was taken to indicate statistical significance.

\section{RESULTS}

\section{Demographic and clinical characteristics}

Of the 1,012 adults diagnosed with pandemic $\mathrm{H} 1 \mathrm{~N} 1$ infection during the study period, 46 patients (4.5\%) were hospitalized and 37 patients (3.7\%) were confirmed to

Table 2. Most common risk factors for study patients

\begin{tabular}{lc}
\hline Comorbidity & Case $(\mathrm{n}=37)$ \\
\hline Chronic pulmonary disease & 8 \\
Metabolic disorder & 5 \\
Chronic cardiovascular disease (excluding & 2 \\
isolated hypertension) & \\
Chronic renal disease & 1 \\
Obesity (body mass index $>30)$ & 1 \\
Pregnancy & 1 \\
Immunosuppressed state (drug, human & 1 \\
immunodeficiency virus, hematological & \\
disorder) & \\
Chronic liver disease & 0 \\
None & 19 \\
\hline
\end{tabular}

have $\mathrm{H} 1 \mathrm{~N} 1$ pneumonia, based on HRCT findings. Five of these 37 patients died (13.5\%).

The demographic and clinical characteristics of the patients are described in Table 1. The mean age of the group was 46.1 years. Twenty-one patients (57\%) were males. Eighteen patients (49\%) had underlying conditions, such as chronic pulmonary disease and metabolic disorders (primarily diabetes mellitus), which were the most commonly noted comorbidities and were risk factors for severe pandemic H1N1 (Table 2). Twelve patients were assigned to the severe group and 25 to the nonsevere group. There was no significant difference in the demographic or clinical data between the two groups. Of the 12 patients in the severe group, eight required mechanical ventilation, five had acute respiratory distress syndrome (ARDS), and four presented with shock. Of the five patients who died, two died of ARDS. The remaining three patients died from sudden cardiac death $(n=1)$, multi-organ failure $(\mathrm{n}=1)$, and exacerbation of underlying chronic obstructive pulmonary disorder $(\mathrm{n}=1)$.

The mean PSI and CURB65 scores were 60 and 0.65 points, respectively. The mean PSI score of the severe group was higher than that of the non-severe group ( $p=0.035)$. However, we found no significant difference in CURB65 values between the groups. The mean time to first dose of antiviral medication was 5.27 days and was not significantly different between the groups ( $p=0.2675$ ).

There was no statistically significant difference in mean leukocyte count, lymphocyte count, or C-reactive protein level between the groups (Table 3). However, the levels of serum aspartate aminotransferase (AST), alanine aminotransferase (ALT), and lactate dehydrogenase (LDH) were significantly higher in the severe group than in the non-severe group ( $p=0.0003,0.0023$, and 0.0002, respectively).

Table 3. Laboratory findings and degree of radiological involvement

\begin{tabular}{lcccc}
\hline Variables & Total & Severe group & Non-severe group & $p$ value \\
\hline Aspartate aminotransferase, U/L & $50.9 \pm 42.4$ & $91.3 \pm 33.1$ & $31.5 \pm 17.3$ & 0.0003 \\
Alanine aminotransferase, U/L & $34.4 \pm 26.8$ & $53.5 \pm 23.2$ & $25.2 \pm 13.8$ & 0.0023 \\
Lactate dehydrogenase, U/L & $620.1 \pm 356.1$ & $945.0 \pm 285.2$ & $464.2 \pm 151.9$ & 0.0002 \\
Leukocytes, $/ \mathrm{mm}^{3}$ & $9,006 \pm 5241$ & $8,393 \pm 3984$ & $9,300 \pm 4015$ & 0.5120 \\
Lymphocytes, $/ \mathrm{mm}^{3}$ & $1,062 \pm 715$ & $1,016 \pm 498$ & $1,085 \pm 574$ & 0.5957 \\
C-reactive protein & $12.3 \pm 17.3$ & $9.4 \pm 6.4$ & $13.7 \pm 15.7$ & 0.4899 \\
Radiological extent, \% & $28.2 \pm 23.5$ & $50.0 \pm 18.6$ & $17.8 \pm 10.0$ & 0.0017 \\
Involved lobes, $\mathrm{n}$ & $4.2 \pm 1.7$ & $5.1 \pm 1.1$ & $3.8 \pm 1.3$ & 0.0301 \\
\hline
\end{tabular}

Values are presented as mean \pm SD. 


\section{HRCT findings}

The HRCT findings are summarized in Table 4. HRCT scans were performed within 15 days of the onset of symptoms. The most common findings were ground-glass attenuation with consolidation $(n=22,60 \%)$, ground glass attenuation alone $(\mathrm{n}=13,35 \%)$, and consolidation alone $(\mathrm{n}=2,5 \%)$. The distribution in the axial plane was predominantly patchy $(\mathrm{n}=31)$, although some patients showed a diffuse distribution $(n=6)$. Patchy distribution of GGA and/or consolidation were seen predominantly at the peripheral subpleural space in 19 patients (51\%) and were random in 12 patients (33\%) (Fig. 1). GGA and/or consolidation were predominant in the lower lung zone in 10 patients (27\%). There was no craniocaudal zonal predominance in the remaining patients. The areas of consolidation were surrounded by GGA in all patients with consolidation and GGA ( $=22)$. "Crazy-paving" appearance, defined by intralobular interstitial thickening in the area of GGA, was observed in 14 patients (38\%) (Fig. 2). Poorly marginated centrilobular nodules were seen in 22 patients (59\%). Bilateral involvement was more common $(\mathrm{n}=30,81 \%)$ than unilateral involvement $(\mathrm{n}=7$, $19 \%)$.

The extent of consolidation and GGA was significantly higher $(p=0.0017)$ in the severe group (average, $50 \% ; 10$ to $90 \%$ ) than in the non-severe group (average, 17.8\%; 5 to $50 \%$ ). Other HRCT findings did not differ significantly between the groups. We found small pleural effusion in seven patients (19\%) and hilar or mediastinal lymph node enlargement in two (5\%). Spontaneous pneumomediastinum was seen in one patient (3\%). No patient had pulmonary thromboembolism (PE).

\section{Correlations between clinical predictors and degree of radiological involvement}

We found significant differences in the PSI score, AST, ALT, and LDH levels between the two groups. The Pearson correlation coefficient was used to evaluate the correlations between these parameters and the degree of radiological involvement. The results indicated that AST, ALT, and LDH levels were correlated with the extent of consolidation and GGA $(p<0.0001,0.0003$, and $<0.0001$, respectively) and the number of involved lobes ( $p=0.663,0.0134$, and 0.0019 , respectively). LDH showed the strongest correlation with the degree of radiological involvement.

\section{DISCUSSION}

Several clinical features were identified as indicative of severe disease early in the $2009 \mathrm{H} 1 \mathrm{~N} 1$ pandemic [7-11]. As age, comorbidity, and timing of the antiviral treatment were described as the most important prognostic factors,

Table 4. Radiological characteristics of pandemic influenza A pneumonia

\begin{tabular}{|c|c|c|c|}
\hline & $\begin{array}{l}\text { Severe group } \\
\qquad(n=12)\end{array}$ & $\begin{array}{l}\text { Non-severe group } \\
\qquad(\mathrm{n}=25)\end{array}$ & $\begin{array}{c}\text { Total } \\
(n=37)\end{array}$ \\
\hline \multicolumn{4}{|l|}{ Pattern } \\
\hline Consolidation & $0(0)$ & $2(8)$ & $2(5)$ \\
\hline Ground-glass attenuation & $2(17)$ & $11(44)$ & $13(35)$ \\
\hline Ground-glass attenuation + consolidation & $10(83)$ & $12(48)$ & $22(60)$ \\
\hline \multicolumn{4}{|l|}{ Distribution } \\
\hline Diffuse & $6(50)$ & $0(0)$ & $6(16)$ \\
\hline Patchy & $6(50)$ & $25(100)$ & $31(84)$ \\
\hline Peripheral & $4(33)$ & $15(60)$ & $19(51)$ \\
\hline Random & $2(17)$ & $10(40)$ & $12(33)$ \\
\hline \multicolumn{4}{|l|}{ Laterality } \\
\hline Unilateral involvement & $3(25)$ & $4(16)$ & $7(19)$ \\
\hline Bilateral involvement & $9(75)$ & $21(84)$ & $30(81)$ \\
\hline Presence of centrilobular nodules & $5(42)$ & $17(68)$ & $22(59)$ \\
\hline Effusion & $5(42)$ & $2(8)$ & $7(19)$ \\
\hline Lymphadenopathy & $0(0)$ & $2(8)$ & $2(5)$ \\
\hline Crazy-paving pattern & $8(67)$ & $6(24)$ & $14(38)$ \\
\hline
\end{tabular}

Values are presented as number (\%). 


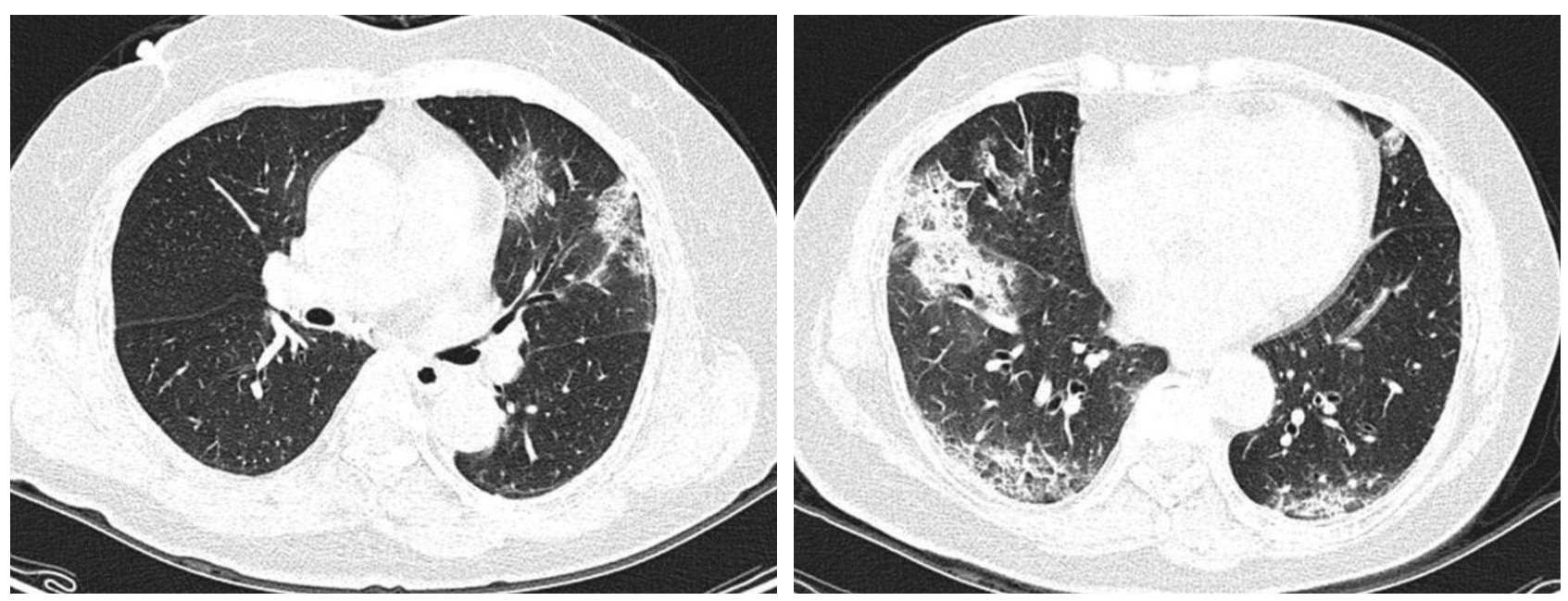

Figure 1. Fifty-five-year-old woman with mild influenza A (H1N1) pneumonia. High resolution computed tomography scans showed multiple patchy areas of consolidation and ground glass attenuation in middle and lower zones of bilateral lungs.
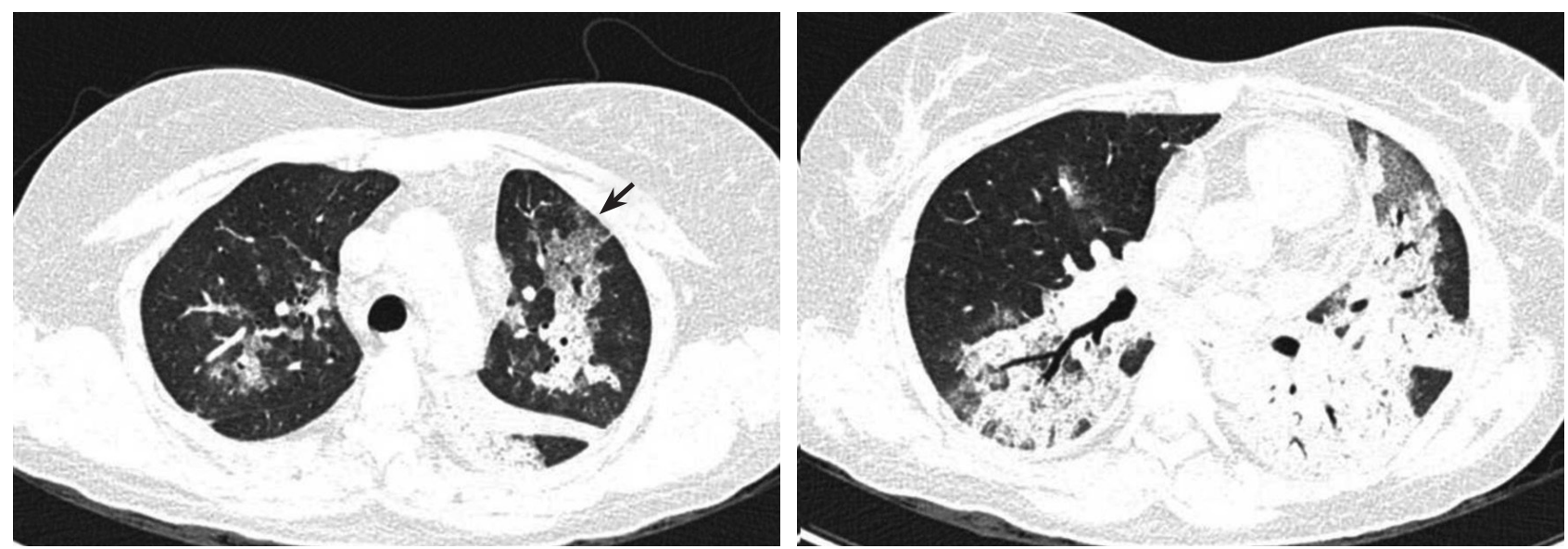

Figure 2. Sixty-one-year-old woman with severe influenza A (H1N1) pneumonia. High resolution computed tomography scans showed diffuse area of consolidation and ground glass attenuation predominantly in lower lung zones. Note crazy-paving appearance (arrows).

we evaluated the clinical significance of these factors and studied their correlations with radiological features in severe pandemic H1N1 pneumonia.

This pandemic had some unusual features of severe disease in comparison with previous influenza pandemics [7-10,15]. While these studies suggested that pandemic $\mathrm{H} 1 \mathrm{~N} 1$ infection caused severe disease in previously healthy and young subjects, other studies $[16,17]$ showed the highest mortality rates in those aged 50 years and older. It is thus unclear whether age is, in fact, a risk factor for severe disease. These conflicting observations may have been due to the presence of comorbidities commonly seen in patients aged 50 and older. However, there was no significant difference in mean age or frequency of comorbidities between the severe and non-severe groups in our study population.

Previous studies [18-20] indicated that prompt antiviral treatment can shorten the duration of seasonal influenza. Maximal benefit was seen when treatment was begun within the first 24-30 hours of onset. Patients with pandemic $\mathrm{H}_{1} \mathrm{~N} 1$ infection in whom antiviral treatment was started within the first 48 hours showed significantly fewer fatalities [16]. Only six of the patients in our study began antiviral treatment within the first 48 hours. Although delayed antiviral treatment was suggested to be a risk factor for pandemic H1N1 pneumonia, our results only showed a trend towards a greater delay in the severe group, with no statistically significant difference in the 
mean time to antiviral treatment between the severe and non-severe groups.

PSI and CURB65 are clinical tools that predict morbidity and mortality in patients with communityacquired pneumonia. CURB65 measures illness activity, whereas PSI was developed to predict the likelihood of mortality [21]. We measured PSI and CURB65 in our study population to evaluate the efficacy of these predictive tools. A significant difference was observed in PSI, but not in CURB65, between the groups, suggesting that PSI may be helpful for predicting the clinical outcome of severe pandemic H1N1 pneumonia. However, as the role of age (the most important factor contributing to PSI) is unclear in pandemic $\mathrm{H} 1 \mathrm{~N} 1$ pneumonia, these results should be interpreted with caution.

We reviewed the laboratory and radiological findings of pandemic H1N1 pneumonia and used univariate analysis to compare these findings with the severity of disease. The two groups differed significantly in serum levels of AST, ALT, and LDH. Although these laboratory findings can be observed in liver disease, there was no evidence of hepatotoxicity from other causes, such as history of drug use, preexisting liver disease, or positive viral markers for acute hepatitis. With regard to radiological findings, the two study groups differed significantly in the extent of GGA or consolidation and the number of involved lobes. We examined correlations between these findings and the degree of radiological involvement to explore the clinical utility of these markers. AST, ALT, and LDH levels were correlated with the degree of radiological involvement, with LDH showing the strongest correlation. These findings may be helpful in managing patients with pandemic H1N1 pneumonia. Although previous studies yielded similar laboratory findings in the severe form of pandemic H1N1 pneumonia, these studies did not correlate the findings with disease severity $[7,8]$. A recent study comparing differences between respiratory failure and non-respiratory failure groups among patients hospitalized with pandemic H1N1 pneumonia [11] showed that multisystem organ failure, lymphopenia, and delayed antiviral treatment were clinical predictors of respiratory failure. However, the study population included all age groups and approximately half of the subjects were younger than 18 years old. Different clinical courses were observed in the $2009 \mathrm{H} 1 \mathrm{~N} 1$ pandemic depending on age group. Infants had the highest hospitalization rates and people aged 50 years and older had the highest mortality rates [16]. Age is thus an important consideration while interpreting the results of the present study.

Our results are consistent with those of recent studies indicating that diffuse or patchy areas of GGA mixed with consolidation were the most common findings on CT scans of H1N1 pneumonia patients [22-26]. Although influenza is not a predisposing factor for pulmonary thromboembolism, $36 \%$ of patients in the severe group were previously reported to have pulmonary thromboembolism [24,27-29]. The patients included in our study underwent routine contrast-enhanced CT scans, performed with an injection rate of $2 \mathrm{~mL} / \mathrm{sec}$ and we found no case of pulmonary thromboembolism. These results may be explained by ethnic differences in the prevalence of PE. The crazy-paving appearance was common (38\%) in our study population. Although this is a non-specific finding, commonly seen in pulmonary alveolar proteinosis, diffuse alveolar hemorrhage, atypical pulmonary infection, or near-drowning [30,31], the high prevalence of this finding among our $\mathrm{H} 1 \mathrm{~N} 1$ pneumonia cases is unique. The crazy-paving appearance was particularly common in the severe group, where the extent of pulmonary involvement was high. In addition to the other indices of severe $\mathrm{H} 1 \mathrm{~N} 1$ pneumonia in the present study, we also found a significantly greater extent of pulmonary involvement with GGA and consolidation among patients with severe pneumonia.

This study had some limitations. First, the small sample size and limited numbers of patients resulted in low statistical power in our analyses. Second, as only patients who visited the emergency room were included in our study population and patients with normal chest radiographs were not included, this may have resulted in over-representation of severe $\mathrm{H} 1 \mathrm{~N} 1$ pneumonia cases and under-representation of non-severe cases presenting with normal findings on chest radiography [24].

In conclusion, the results of the present study indicated that higher serum levels of AST, ALT, and LDH were significantly associated with severe pandemic $\mathrm{H} 1 \mathrm{~N} 1$ pneumonia. The most common CT findings were bilateral subpleural patchy or diffuse areas of GGA and consolidation. Patients with severe illness had a significantly greater extent of lung involvement, based on a higher prevalence of crazy-paving appearance on HRCT scans. A combination of clinical and HRCT indicators may therefore aid in predicting the clinical course and outcome of pandemic H1N1 pneumonia. 


\section{Conflict of interest}

No potential conflict of interest relevant to this article was reported.

\section{Acknowledgments}

This work was supported for two years by Pusan Nationl University Research Grant.

\section{REFERENCES}

1. Centers for Disease Control and Prevention (CDC). Swine influenza A (H1N1) infection in two children: Southern California, March-April 2009. MMWR Morb Mortal Wkly Rep 2009;58:400-402.

2. Centers for Disease Control and Prevention (CDC). Update: influenza activity: United States, August 30-October 31, 2009. MMWR Morb Mortal Wkly Rep 2009;58:1236-1241.

3. Centers for Disease Control and Prevention (CDC). Hospitalized patients with novel influenza A ( $\mathrm{H} 1 \mathrm{N1}$ ) virus infection: California, April-May, 2009. MMWR Morb Mortal Wkly Rep 2009;58:536541.

4. Human infection with pandemic $\mathrm{A}(\mathrm{H} 1 \mathrm{N1}) 2009$ influenza virus: clinical observations in hospitalized patients, Americas, July 2009 - update. Wkly Epidemiol Rec 2009;84:305-308.

5. Epidemiological summary of pandemic influenza A (H1N1) 2009 virus: Ontario, Canada, June 2009. Wkly Epidemiol Rec 2009;84:485-491.

6. Centers for Disease Control and Prevention (CDC). Patients hospitalized with 2009 pandemic influenza A (H1N1): New York City, May 2009. MMWR Morb Mortal Wkly Rep 2010;58:14361440.

7. Perez-Padilla R, de la Rosa-Zamboni D, Ponce de Leon S, et al. Pneumonia and respiratory failure from swine-origin influenza A (H1N1) in Mexico. N Engl J Med 2009;361:680-689.

8. Rello J, Rodriguez A, Ibanez P, et al. Intensive care adult patients with severe respiratory failure caused by influenza $A\left(\mathrm{H}_{1} \mathrm{~N} 1\right) \mathrm{v}$ in Spain. Crit Care 2009;13:R148.

9. Kumar A, Zarychanski R, Pinto R, et al. Critically ill patients with 2009 influenza $\mathrm{A}\left(\mathrm{H}_{1} \mathrm{~N} 1\right)$ infection in Canada. JAMA 2009;302:1872-1879.

10. Dominguez-Cherit G, Lapinsky SE, Macias AE, et al. Critically Ill patients with 2009 influenza $\mathrm{A}(\mathrm{H} 1 \mathrm{~N} 1)$ in Mexico. JAMA 2009;302:1880-1887.

11. Chien YS, Su CP, Tsai HT, et al. Predictors and outcomes of respiratory failure among hospitalized pneumonia patients with 2009 H1N1 influenza in Taiwan. J Infect 2010;60:168-174.

12. Mandell LA, Wunderink RG, Anzueto A, et al. Infectious Diseases Society of America/American Thoracic Society consensus guidelines on the management of community- acquired pneumonia in adults. Clin Infect Dis 2007;44 Suppl 2:S27-S72.

13. Fine MJ, Auble TE, Yealy DM, et al. A prediction rule to identify low-risk patients with community-acquired pneumonia. N Engl J Med 1997;336:243-250.

14. Lim WS, van der Eerden MM, Laing R, et al. Defining community acquired pneumonia severity on presentation to hospital: an international derivation and validation study. Thorax 2003;58:377-382.

15. Fisman DN, Savage R, Gubbay J, et al. Older age and a reduced likelihood of $2009 \mathrm{H} 1 \mathrm{~N} 1$ virus infection. N Engl J Med 2009;361:2000-2001.

16. Louie JK, Acosta M, Winter K, et al. Factors associated with death or hospitalization due to pandemic 2009 influenza A(H1N1) infection in California. JAMA 2009;302:1896-1902.

17. Vaillant L, La Ruche G, Tarantola A, Barboza P. Epidemiology of fatal cases associated with pandemic H1N1 influenza 2009. Euro Surveill 2009;14:19309.

18. Randomised trial of efficacy and safety of inhaled zanamivir in treatment of influenza A and B virus infections: the MIST (Management of Influenza in the Southern Hemisphere Trialists) Study Group. Lancet 1998;352:1877-1881.

19. Nicholson KG, Aoki FY, Osterhaus AD, et al. Efficacy and safety of oseltamivir in treatment of acute influenza: a randomised controlled trial. Neuraminidase Inhibitor Flu Treatment Investigator Group. Lancet 2000;355:1845-1850.

20. Jefferson T, Demicheli V, Rivetti D, Jones M, Di Pietrantonj C, Rivetti A. Antivirals for influenza in healthy adults: systematic review. Lancet 2006;367:303-313.

21. Chalmers JD, Singanayagam A, Hill AT. Predicting the need for mechanical ventilation and/or inotropic support for young adults admitted to the hospital with community-acquired pneumonia. Clin Infect Dis 2008;47:1571-1574.

22. Kim EA, Lee KS, Primack SL, et al. Viral pneumonias in adults: radiologic and pathologic findings. Radiographics 2002;22 Spec No:S137-S149.

23. Lee CW, Seo JB, Song JW, et al. Pulmonary complication of novel influenza A (H1N1) infection: imaging features in two patients. Korean J Radiol 2009;10:531-534.

24. Agarwal PP, Cinti S, Kazerooni EA. Chest radiographic and CT findings in novel swine-origin influenza A (H1N1) virus (S-OIV) infection. AJR Am J Roentgenol 2009;193:1488-1493.

25. Ajlan AM, Quiney B, Nicolaou S, Muller NL. Swine-origin influenza A ( $\mathrm{H} 1 \mathrm{N1}$ ) viral infection: radiographic and CT findings. AJR Am J Roentgenol 2009;193:1494-1499.

26. Marchiori E, Zanetti G, Hochhegger B, et al. High-resolution computed tomography findings from adult patients with Influenza A (H1N1) virus-associated pneumonia. Eur J Radiol 2010;74:93-98.

27. Centers for Disease Control and Prevention (CDC). Intensivecare patients with severe novel influenza A (H1N1) virus infection: Michigan, June 2009. MMWR Morb Mortal Wkly Rep 
2009;58:749-752.

28. Schultz MJ, Haitsma JJ, Zhang H, Slutsky AS. Pulmonary coagulopathy as a new target in therapeutic studies of acute lung injury or pneumonia: a review. Crit Care Med 2006;34:871-877.

29. van Wissen M, Keller TT, Ronkes B, et al. Influenza infection and risk of acute pulmonary embolism. Thromb J 2007;5:16.
30. Johkoh T, Itoh H, Muller NL, et al. Crazy-paving appearance at thin-section CT: spectrum of disease and pathologic findings. Radiology 1999;211:155-160.

31. Kim KI, Lee KN, Tomiyama N, et al. Near drowning: thinsection CT findings in six patients. J Comput Assist Tomogr 2000;24:562-566. 